\title{
The status of GEO 600
}

\author{
H Grote (for the LIGO Scientific Collaboration)
}

Max-Planck-Institut für Gravitationsphysik (Albert-Einstein-Institut) und Universität Hannover, Callinstr. 38, D-30167 Hannover, Germany

E-mail: hartmut.grote@aei.mpg.de

Received 26 October 2007, in final form 17 December 2007

Published 15 May 2008

Online at stacks.iop.org/CQG/25/114043

\begin{abstract}
The GEO 600 gravitational wave detector located near Hannover in Germany is part of the LSC network of gravitational wave observatories. Since January 2006 the GEO 600 detector has participated in the S5 LSC science run and acquired sensitive and well-characterized science data with a high duty cycle. Until 1 October 2007, 415 days of science data with an average peak sensitivity of better than $3 \times 10^{-22} \mathrm{~Hz}^{-1 / 2}$ have been collected. In this paper, we give a brief overview of GEO 600 and describe activities in the period between January 2006 and October 2007. Plans for the near and medium future are briefly discussed.
\end{abstract}

PACS numbers: $04.80 . \mathrm{Nn}, 95.55 . \mathrm{Ym}$, 95.75.Kk

(Some figures in this article are in colour only in the electronic version)

\section{Introduction}

The international network of large-scale laser-interferometric gravitational wave (GW) detectors currently consists of LIGO [1], TAMA300 [2], Virgo [3] and GEO 600 [4].

A simplified layout of the GEO 600 interferometer is shown in figure 1. The light of a master-slave laser systems is filtered by two sequential input mode cleaners (MC1 and MC2), ring cavities of about $8 \mathrm{~m}$ round-trip length consisting of three mirrors each. The stabilized and filtered light is then injected through the power-recycling mirror (MPR) into the main interferometer, formed by the Michelson interferometer with single folded arms of $600 \mathrm{~m}$ length. The operating point is chosen to be the dark fringe, thus only signal sidebands and control sidebands leave the interferometer towards the output port, which hosts the signal-recycling mirror (MSR). The GW signal is finally read out by using a radio-frequency heterodyne technique. 


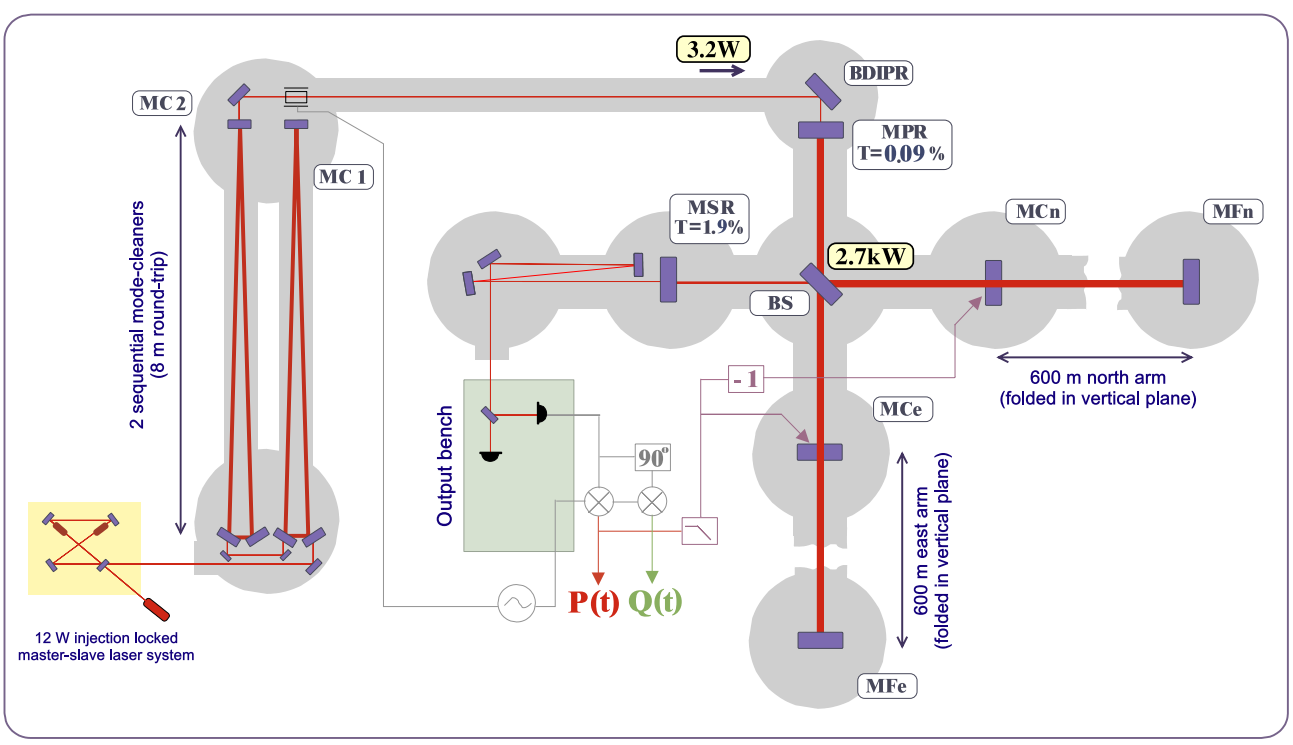

Figure 1. Simplified optical layout of the GEO 600 detector. A more detailed description is given in the text.

\section{Advanced techniques of GEO 600}

During the commissioning of GEO 600 many challenges were encountered. In this section, we briefly describe some of them which are related to the advanced techniques of GEO 600: triple suspensions with monolithic stages, electro-static actuators and signal-recycling.

In order to minimize effects from thermal noise in the suspensions, the core optics of GEO 600 are suspended from triple cascaded pendulums with quasi monolithic last stages made of thin fused silica fibres [5]. These have low dissipation in flexure and their attachment to the test mass eliminates friction at that point. Loss angles of various test mass internal modes have been measured to be as low as $2.5 \times 10^{-7}$ [6]. To avoid an instability of the control system, the mechanical quality factor $Q$ of transversal fibre eigenmodes (violin modes) of the fused silica fibres had to be reduced without affecting the pendulum $Q$. This was realized by a novel technique employing damping, mainly of the first- and second-order violin modes, via a coating of amorphous Teflon at the relevant fibre sections [7].

Actuators are present at each pendulum stage, which allows us to hierarchically split the longitudinal and alignment control signals according to the range and to the frequency band of the individual actuators $[8,9]$. Short-range, fast corrections are applied directly to the mirror, with progressively longer range and lower frequency components being applied at the stages further up the suspension.

While standard coil magnet combinations are used for actuation at the top and intermediate pendulum stages, electro-static drives (ESD) are used as fast actuators directly acting onto the test masses. By using ESDs instead of a standard coil magnet system, it can be avoided to attach magnets to the test masses, which could degrade the thermal noise properties of the mirror. A fringing field design is employed with the curved field-lines from interleaving combs of electrodes at different voltages penetrating the dielectric test mass. At the design operating point, where the gap between the drive and test mass is about the same as the gap between the fingers of the alternating electrode combs, i.e. $2.5 \mathrm{~mm}$, the unidirectional force $F$ 
acting on the test mass is given by

$$
F=U^{2} \cdot \varepsilon \cdot \varepsilon_{r} \cdot d^{-3 / 2} \cdot a,
$$

where $U$ is the voltage difference, $\varepsilon$ and $\varepsilon_{r}$ are the dielectric constant and the relative dielectric constant of the test mass substrate, $d$ is the distance between the test mass and the ESD and $a$ is a constant geometry factor depending on the electrode pattern design [10]. A potential problem of electro-static actuators is charged particles deposited on the test-mass, facing the electrode pattern of the ESD. With the presence of such positive or negative charges, a force term is added to the expression in equation (1), that is linear dependent on the voltage $U$. Such a term changes the test-mass response to the drive voltage, and thus has potential effects on control loop gains and calibration factors. However, within 6 years of operation, such an effect has been observed only once, in late 2006. This event leads to a more detailed investigation and the application of a method to remove charges from the test-masses by illumination with UV-light [11].

GEO 600 is the first large-scale GW detector taking advantage of dual-recycling, which is the combination of power-recycling and signal-recycling. While power-recycling increases the storage time of the carrier light, signal-recycling allows shaping of the detector response and increases the sensitivity in a certain frequency band. Signal-recycling provides a variety of operation modes such that the detector response can be adjusted to optimally match astrophysical targets. In order to maximize the science contribution of GEO 600 within the network of the LSC detectors it is operated with detuned signal-recycling, i.e. the signalrecycling cavity is chosen to be off resonance for the carrier light. For the S5 run a detuning of $550 \mathrm{~Hz}$ was chosen, resulting in the fact that the peak sensitivity of the detector is located around this frequency.

In the detuned signal-recycling configuration, the signal-recycling cavity is not resonant for the carrier light and has different response functions for the upper- and lower signalrecycling sidebands. This is the cause of a strong imbalance of the detected control and signal sidebands which leads to several potential disadvantages compared to tuned signal-recycling, including increased coupling of technical noise [12] to the detector output. Another speciality of detuned signal-recycling is the fact that the distribution of the GW signal onto the two orthogonal output quadratures $P(t)$ and $Q(t)$ depends on the signal frequency. These two signals are optimally combined, using a maximum likelihood method, resulting in a single GW channel, $h(t)$, providing best signal-to-noise ratio at all frequencies [13].

Recently the operation of tuned signal-recycling was demonstrated in GEO 600 and is considered as an optional configuration for the medium-term future (see also [12]). In addition to this we recently succeeded in first experiments replacing the heterodyne readout method by a homodyne scheme, in which the Michelson is operated with a dedicated offset from the dark fringe, in order to generate a local oscillator beam of carrier light at the dark port. We are testing a scheme, in which no output modecleaner is used. In order to dominate the light power at the dark port with the homodyne local oscillator, the radio-frequency modulation sidebands are reduced by a factor of 10 after lock acquisition [14]. Stable detector operation was achieved for tuned as well as detuned signal-recycling states. The investigation of these operating modes is ongoing.

\section{The noise budget and glitch rates of GEO 600}

Figure 2 shows the actual noise budget of GEO 600 close to the end of the S5 LSC science run. The largest noise sources above a few $100 \mathrm{~Hz}$ are shot noise, RF noise and oscillator phase noise (explained below), and dark noise, which is the electronic noise of the main 


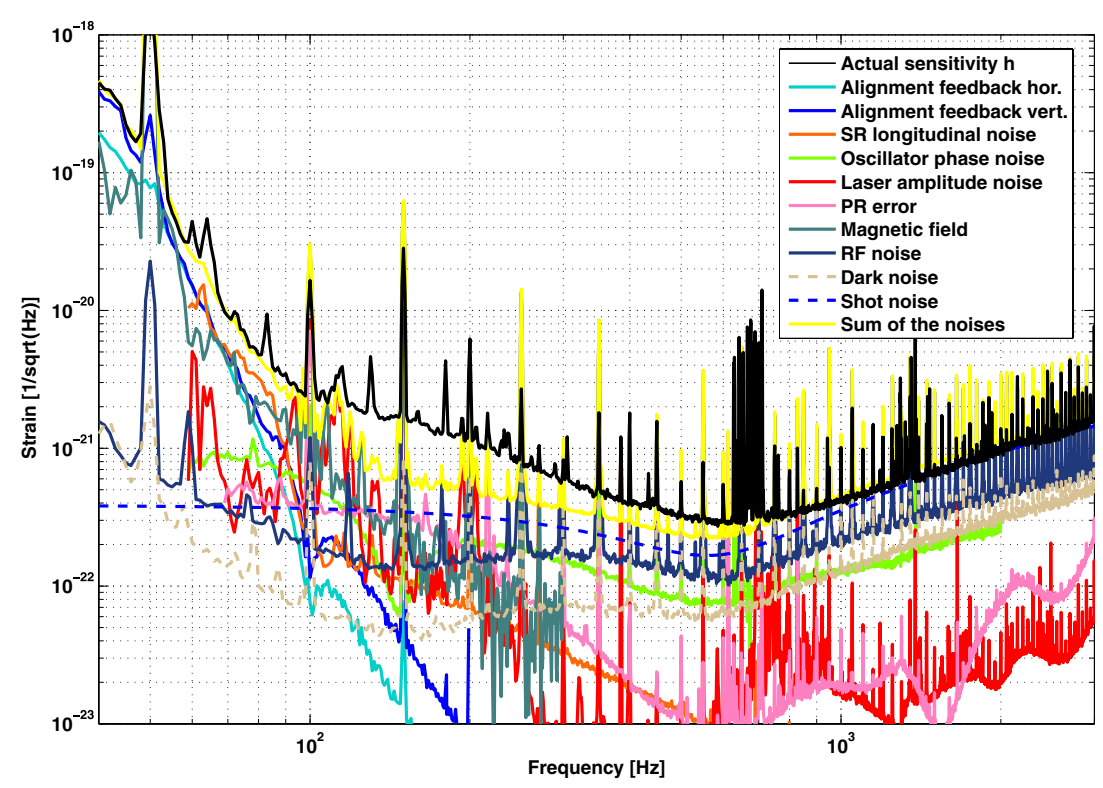

Figure 2. Noise projection of various signals for a time close to the end of the S5 LSC science run. At high frequencies the sensitivity is mainly limited by shot noise, while at low frequencies (below $100 \mathrm{~Hz}$ ) feedback noise and magnetic noise are dominating. Feedback noise is shown here for the signal-recycling (SR) mirror longitudinal control and the two alignment degrees of freedom of the Michelson differential control. Laser amplitude noise contributes a bit around $120 \mathrm{~Hz}$. In the region between $100 \mathrm{~Hz}$ and $500 \mathrm{~Hz}$ a discrepancy between the uncorrelated sum of all noise projections and the actual observed sensitivity is found.

photodetector in case no light is incident on it. At low frequencies (below $100 \mathrm{~Hz}$ ) feedback noise and magnet field noise are the dominating noise contributions. Feedback noise originates from control loops, in this case these are the signal-recycling mirror longitudinal control, and the two degrees of freedom of the Michelson end mirror alignment control. Laser amplitude noise dominates ' $h$ ' around a resonant peak of the laser amplitude noise transfer function at $120 \mathrm{~Hz}$. In the region between $100 \mathrm{~Hz}$ and $500 \mathrm{~Hz}$ a discrepancy between the uncorrelated sum of all noise projections and the actual observed sensitivity is found. We investigated possible sources of limiting noise in this frequency band. Examples of noise sources that were investigated in 2007 more deeply than before include

- laser frequency noise,

- oscillator phase noise,

- scattered light noise.

These are briefly described in the following.

In GEO 600 the power-recycling (PR) cavity is used as frequency reference. A control loop with a bandwidth of $25 \mathrm{kHz}$ adjusts the input laser frequency to be resonant in the PR cavity. Laser frequency noise couples to the detector output by the small but nonzero length difference of the long arms, which is used to pass RF-modulation sidebands to the dark port. Provided the loop stabilizing the laser frequency has sufficient gain, the laser frequency noise input to the power recycling cavity is determined by the quality of the sensor comparing the incident light with the light resonant within the PR cavity. To investigate the sensing chain, we implemented a second photodiode assembly similar to that normally used. This photodiode can be regarded as an out-of-loop measurement, as far as the detection process on 
the photodiode and beyond is concerned. We showed that the noise level of this out-of-loop diode is very close to the nearly shot noise limited level of the in-loop diode. This is entirely consistent with the fact that the in-loop diode, and the signal processing beyond, do not add any excess noise. For the projection of laser frequency noise to ' $h$ ', we use the larger one of either the in-loop signal or the shot noise limit of the detector, evaluated for each frequency. This projection is shown by the trace labelled 'PR error' in figure 2 . With this projection, the laser frequency noise is a factor 5 below the current sensitivity (labelled ' $h$ ' in figure 2), from 100 to $200 \mathrm{~Hz}$.

Currently we use two different ways to project phase noise of the Michelson RFmodulation path. In the normal running condition, we have a monitor channel that measures phase noise of the crystal oscillator that drives the EOM and the local oscillator path. A part of the signal in the local oscillator path is split off and a separate crystal oscillator is phase-locked onto this. The PLL (phase locked loop) used for this has a bandwidth of about $1 \mathrm{~Hz}$. The beat signal between the two oscillators is regularly recorded and used for projection to ' $h$ ' [15]. Figure 2 shows this contribution as 'oscillator phase noise'. We recently also employed another test for phase noise, including a large fraction of the RF electronics. In this scheme, we loosely speaking replaced the interferometer by a wire. This means that we fed the RF-sinewave going to the EOM (electro-optic modulator) for the normal modulation directly to the mixer input with appropriate amplitude and phase. We chose the level and phase at the mixer input to be the same as in the normal locked case. This scheme does not only take oscillator phase noise into account, but also RF phase- and amplitude noise of all components in the modulation- and demodulation path. The resulting projection is shown by the trace labelled 'RF noise' in figure 2, and can be regarded as a lower limit of RF phase- and amplitude noise in the current setup.

Back-scattering from optical components outside the vacuum system can relatively easy be diagnosed in cases where attenuation filters can be implemented in the beam path [16]. Except for the output port, this had been done during 2006, with no dominant scattering source found. We recently could perform such a test for the output port as well, using a new device, acting as a phase modulator of the passing light [17]. A fused-silica substrate with a large aperture of approx. Ten centimetre was placed as the first optical component on the beam leaving the vacuum system at the output port. A piezoelectric actuator attached to the substrate excites an eigenmode of the substrate at about $24 \mathrm{kHz}$, thus creating a modulated optical pathlength within the substrate. With this technique, any beam caused by scattering, that passes the modulator again on its way back to the interferometer, no longer beats with the single-pass beam at frequencies within the detection band. We could show that this method suppresses artificially forced scattering pretty well. Using the device at the output port did not change the detector sensitivity curve at any frequency, showing that GEO 600 is currently not limited by back-scattering from this port.

To investigate possible scattering sources within the vacuum system we excited vacuum tanks with mechanical vibrations, which mainly tests for scattering of components which are directly connected to the tank. For example, this is the case for auxiliary pick-off mirrors and parts of the suspension system (e.g. mirror catchers). In the north end tank, a scattering source was identified by this method and removed. Scattering coupling to ' $h$ ' was caused there by a copper ring mounted behind the end-mirror. This ring was formerly used as an electrostatic actuator for ring-down measurements of the mirror substrate. Mechanical excitation of the vacuum tanks in the central building area did not yet reveal any scattering source, but investigations are ongoing.

Besides the investigation of limiting noise sources, we also spent some effort in a further reduction of the glitch rate of GEO 600. A low glitch rate is particularly desirable for 

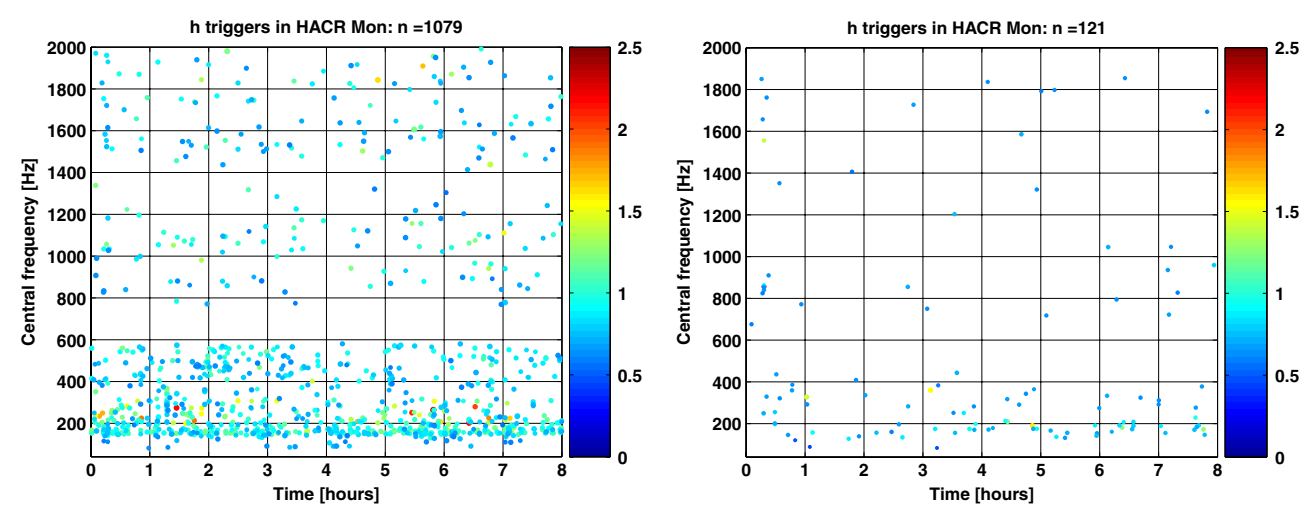

Figure 3. Time-frequency plots of glitch events in the detector output $H$. The left graph is typical for a time series from summer 2006. The right one shows a time series from September 2007. Each dot represents a glitch, with the dot colour encoding the signal-to-noise ratio of the glitch on a logarithmic scale. Measures that reduced the glitch rate are described in the text.

burst searches, where event candidate lists are generated from generic events not being modelled by particular signal templates. A comparison of glitch rates between the LIGO, Virgo and GEO detectors in September 2006 revealed a glitch rate of GEO that was around the average of the other detectors. Since then we further reduced the glitch rate. The exchange of main supply transformers for better balanced types reduced fluctuating magnetic field contributions to ' $h$ ', and also reduced the glitch rate. The removal of the scattering source behind mirror MFn described above contributed to the glitch reduction as well. A third improvement was a complete rebuilt of the RF-modulation system for the power-recycling loop. Figure 3 shows the comparison of glitch rates for a typical time series of summer 2006 and of September 2007. The latter shows an average glitch rate of about 1 glitch within 4 min.

\section{The S5 LSC science run}

At the end of the year 2005, the three LIGO detectors started the S5 LSC science run. In contrast to previous science runs which had durations of up to a few months, S5 lasted about two years. From 21 January 2006, the GEO 600 detector has participated in this long data taking activity, which ended on 1 October 2007. The participation of GEO 600 in S5 can be split into three different periods:

- night- and weekend-mode, period 1: 21/01/2006 to 01/05/2006,

- 24/7-mode: 01/05/2006 to 16/10/2006,

- night- and weekend-mode, period 2: 16/10/2006 to 01/10/2007.

Each of these periods is briefly described in the following subsections.

The strain sensitivities of the four LSC detectors in the network are shown in the upper subplot of figure 4. The lower subplot shows the corresponding displacement sensitivities. For the GEO detector, a displacement of the far mirror couples twice as much into $h(t)$ as a displacement of the near mirror. In this plot, the sensitivity to a displacement of those mirrors, MFe and MFn in figure 1, is given. 

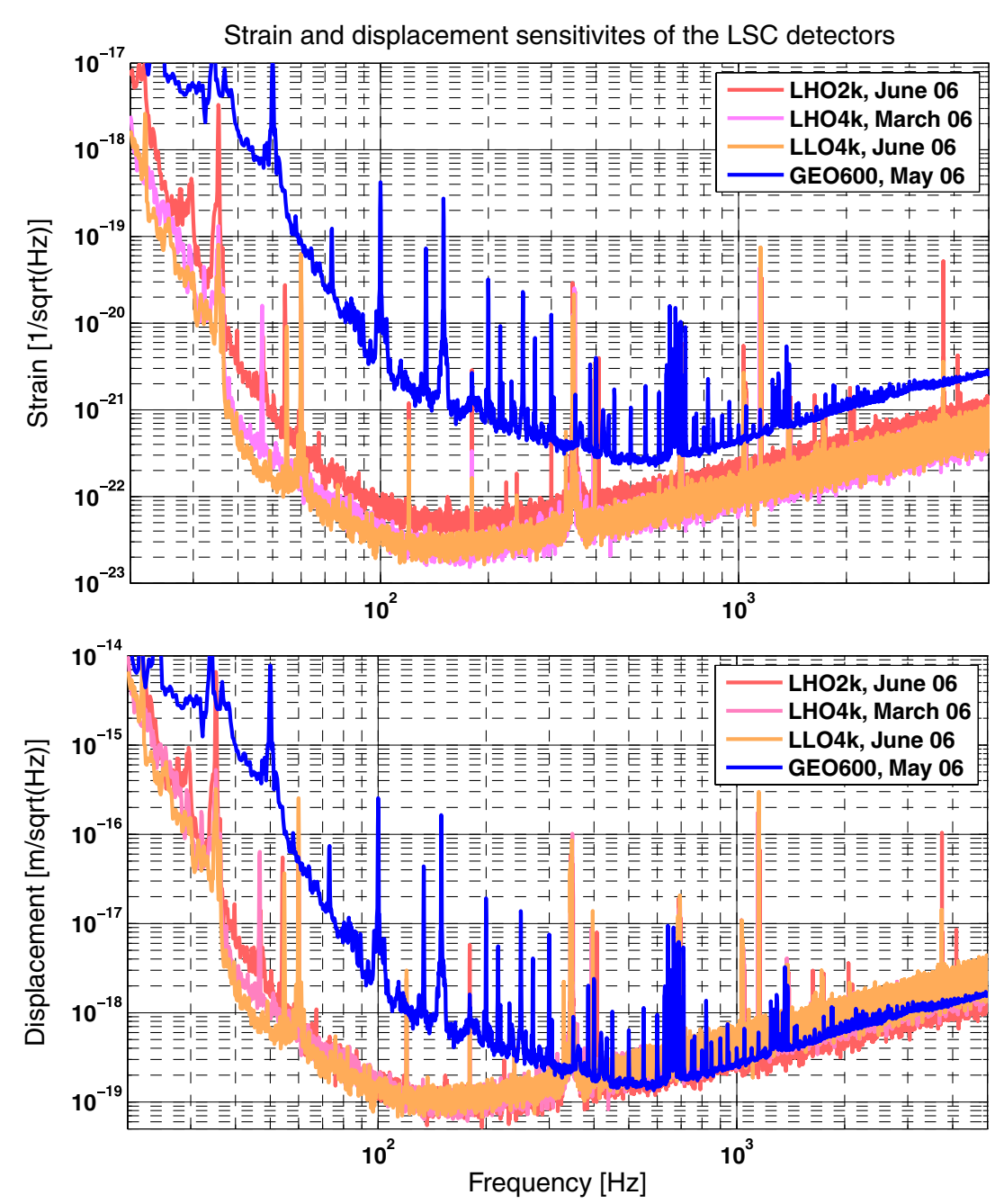

Figure 4. Strain and displacement sensitivities of the LSC detectors during the S5 science run. Shown are the LIGO interferometers $(\mathrm{LHO} 2 \mathrm{k}=$ Hanford $2 \mathrm{~km}$, LHO $4 \mathrm{k}=4 \mathrm{~km}$, LLO $4 \mathrm{k}=$ Livingston $4 \mathrm{~km}$ ) and the GEO 600 detector.

\subsection{Night- and weekend-mode, period 1}

In this first section of the S5 run, science data have been taken during nights and at weekends, while the day time was dedicated to commissioning work, with care being taken to avoid disrupting the science periods. This commissioning work was mainly focused on gaining a more detailed understanding of the instrument performance and increasing the data quality. Strong efforts were put towards investigating glitches, to identify them and achieve their reduction in several interferometer channels. The highest priorities were to maintain a good calibration and characterize the science data taken during nights and weekends.

\subsection{4/7-mode}

In the period from 1 May to 6 October 2007, GEO 600 was operated in the so-called 24/7mode, meaning that we tried to collect science data $24 \mathrm{~h}$ per day, 7 days a week, seeking for a 
Table 1. A summary of the accumulated science time and the science time duty cycle of the GEO 600 detector during the S5 LSC science run so far (21 January 2006 to 1 October 2007).

\begin{tabular}{llll}
\hline Period & $\begin{array}{l}\text { Duration } \\
\text { (days) }\end{array}$ & $\begin{array}{l}\text { Accumulated } \\
\text { science time (days) }\end{array}$ & $\begin{array}{l}\text { Science time } \\
\text { duty cycle (\%) }\end{array}$ \\
\hline N\&W-mode 1 & 100 & 46.5 & 46.5 \\
24/7-mode & 168 & 152.4 & 90.7 \\
N\&W-mode 2 & 350 & 222.6 & 63.6 \\
Total & 618 & 421.5 & 68.2 \\
\hline
\end{tabular}

science time duty cycle as high as possible. Only very short maintenance periods took place for re-measuring noise transfer functions. Overall in 24/7-mode an instrumental duty cycle of about $95 \%$ and a science time duty cycle of more than $90 \%$ were achieved.

An average peak sensitivity of better than $3 \times 10^{-22} \mathrm{~Hz}^{-1 / 2}$ was obtained. Detailed information about the performance of GEO 600 is generated automatically and stored on websites called GEO 600 reports, which contain comprehensive information about sensitivity, data quality, calibration and glitchiness of the instrument.

\subsection{Night- and weekend-mode, period 2}

In a trade-off-decision in October 2006 the following three points were taken into account:

- the necessity for maintenance of the infrastructure,

- the chance to improve the sensitivity with further commissioning work, in order to maximize the science impact of GEO 600 during off-line times of the other detectors in the network,

- the continuation of acquisition of science data at the current sensitivity in coincidence with the LIGO detectors.

With input from the LSC data analysis groups, the LSC operations committee and a detailed benefit/risk analysis of the GEO 600 commissioning team, the decision was made to take the GEO detector out of the 24/7 mode (see also section 5). On 16 October 2006, GEO 600 started a second period of night- and weekend-mode. The beginning of this period was dedicated mainly to non-invasive investigations necessary for future planning of the detector operation. Then in 2007 the work shifted towards invasive hardware changes in order to increase the reliability of the instrument and to reduce its glitch rate. Furthermore, investigations of the limits to the sensitivity were performed in this period, as described in section 3 .

\subsection{Summary of $S 5$}

Overall GEO 600 collected about 415 days of well calibrated and characterized science data in the period between January 2006 and October 2007. Table 1 gives an overview of the accumulated science time and duty cycle for the three different periods of S5.

\section{Future plans}

Since the three LIGO detectors and VIRGO are shutting down their interferometers for installation and commissioning of enhanced LIGO and Virgo+ in 2008, it was decided that GEO 600 will have a long science data taking to cover this period. This program is now active under the name astrowatch. The main focus of this period will be to run GEO 600 in 
data taking mode with a high duty cycle. However within this period, we will still allow for commissioning work at a low level, that involves necessary maintenance work, but can involve sensitivity improvements, whenever a risk/benefit analysis is in favour of such work.

In addition to this, we intend to perform further experiments of the dc-readout scheme for the tuned signal-recycling state. This configuration seems also to be promising for the application of squeezed light, which is considered to be part of the medium- to long term future of GEO 600, namely GEO-HF.

\section{Acknowledgments}

The authors are grateful for support from the Science and Technology Facilities Council (STFC), the University of Glasgow in the UK, the Bundesministerium für Bildung und Forschung (BMBF) and the state of Lower Saxony in Germany.

\section{References}

[1] Sigg D et al 2004 Commissioning of LIGO detectors Class. Quantum Grav. 21 S409-S415

[2] Takahaschi R (the TAMA Collaboration) 2004 Status of TAMA300 Class. Quantum Grav. 21 S403-S408

[3] Acemese F et al 2004 Status of VIRGO Class. Quantum Grav. 21 S385-S394

[4] Willke B et al 2004 Status of GEO 600 Class. Quantum Grav. 21 S417-S423

[5] Plissi M et al 2000 GEO 600 triple pendulum suspension system: seismic isolation and control Rev. Sci. Instrum. 71 2539-45

[6] Smith R et al 2004 Mechanical quality factor measurements of monolithically suspended fused silica test masses of the GEO600 gravitational-wave detector Class. Quantum Grav. 21 1091-8

[7] Gossler S et al 2004 Damping and tuning of the fibre violin modes in monolithic silica suspension Class. Quantum Grav. 21 923-33

[8] Grote H et al 2004 Alignment control of GEO 600 Class. Quantum Grav. 21 S441-S449

[9] Grote H et al 2005 The status of GEO 600 Class. Quantum Grav. 22 S193-S198

[10] Grote H 2003 Making it work: second generation interferometry in GEO 600! PhD Thesis Hannover

[11] Hewitson M et al 2008 Charge measurement and mitigation for the main test-masses of the GEO 600 Gravitational Wave Observatory Class. Quantum Grav. submitted

[12] Hild S et al 2007 Demonstration and comparison of tuned and detuned signal recycling in a large-scale gravitational wave detector Class. Quantum Grav. 24 1513-23

[13] Hewitson M et al 2005 Optimal time-domain combination of the two calibrated output quadratures of GEO 600 Class. Quantum Grav. 22 4253-4261

[14] Hild S et al 2008 Homodyne and heterodyne readout of a signal-recycled GW-detector Class. Quantum Grav. submitted

[15] Smith J R et al 2006 Projection of technical noise for interferometric gravitational-wave detectors Class. Quantum Grav. 23 527-537

[16] Hild S et al 2006 Towards gravitational wave astronomy: commissioning and characterization of GEO600 J. Phys.: Conf. Ser. 32 66-73

[17] Lück H et al 2008 Title TBD Class. Quantum Grav. submitted 